\title{
Immunobiology of Steroid-Unresponsive Severe Asthma
}

\author{
Courtney Lynn Marshall ${ }^{1,2}$, Kosovare Hasani ${ }^{1}$ and Neeloffer Mookherjee ${ }^{1,2 *}$ \\ ${ }^{1}$ Department of Internal Medicine, Manitoba Center of Proteomics and Systems Biology, University of Manitoba, Winnipeg, \\ MB, Canada, ${ }^{2}$ Department of Immunology, Rady Faculty of Health Sciences, University of Manitoba, Winnipeg, MB, Canada
}

Asthma is a heterogeneous respiratory disease characterized by airflow obstruction, bronchial hyperresponsiveness and airway inflammation. Approximately $10 \%$ of asthma patients suffer from uncontrolled severe asthma (SA). A major difference between patients with SA from those with mild-to-moderate asthma is the resistance to common glucocorticoid treatments. Thus, steroid-unresponsive uncontrolled asthma is a hallmark of SA. An impediment in the development of new therapies for SA is a limited understanding of the range of immune responses and molecular networks that can contribute to the disease process. Typically SA is thought to be characterized by a Th2-low and Th17-high immunophenotype, accompanied by neutrophilic airway inflammation. However, Th2-mediated eosinophilic inflammation, as well as mixed Th1/Th17-mediated inflammation, is also described in SA. Thus, existing studies indicate that the immunophenotype of SA is diverse. This review attempts to summarize the interplay of different immune mediators and related mechanisms that are associated with airway inflammation and the immunobiology of SA.

Amanda Louise Tatler, University of Nottingham, United Kingdom

Reviewed by:

Viviana Marin-Esteban Université Paris-Saclay, France Dawn C. Newcomb, Vanderbilt University, United States

*Correspondence:

Neeloffer Mookherjee neeloffer.mookherjee@umanitoba.ca

Specialty section:

This article was submitted to

Asthma,

a section of the journal

Frontiers in Allergy

Received: 31 May 2021 Accepted: 09 August 2021 Published: 27 August 2021

Citation:

Marshall $C L$, Hasani $K$ and Mookherjee N (2021) Immunobiology of Steroid-Unresponsive Severe Asthma. Front. Allergy 2:718267. doi: 10.3389/falgy.2021.718267

\section{INTRODUCTION}

Asthma is a complex, respiratory disease characterized by airway inflammation and bronchoconstriction, which make it difficult to breathe. Asthma affects $\sim 300$ million people worldwide (1). There are heterogeneous clinical symptoms with varying degrees of response to therapy in asthma. Inhaled corticosteroid (ICS) is a common therapy for asthma, to which controllers such as a long-acting $\beta 2$ agonist (LABA) are added if required, and if these fail oral corticosteroids are also added $(2,3)$. Approximately $10 \%$ of asthma patients do not respond to available steroid treatments (2). In 2014, a task force of ERS/ATS defined severe asthma (SA) as "asthma which requires treatment with high dose inhaled corticosteroids (ICS) plus a second controller (and/or systemic corticosteroids) to prevent it from becoming 'uncontrolled', or which remains 'uncontrolled' despite this therapy" (2). Although patients with SA make up a small proportion of asthma patients, this subgroup accounts for more than $50 \%$ of direct and indirect healthcare costs associated with asthma (1). Research in the last three decades has shown that there are multiple phenotypes or subgroups in SA, with differences in clinical symptoms and molecular profiles. There is also a prominent sex-related disparity, as SA disproportionally affects adult females compared to males. Some studies demonstrate that almost $2 / 3 \mathrm{rd}$ of severe asthmatics are females (4). Resistance to corticosteroids can be attributed to a variety of components, from genetic variability to various molecular factors such as defective glucocorticoid receptor (GR) function with increased expression of the non-responsive isoform of $\mathrm{GR} \beta$, different transcription factor and signaling pathways, as well as specific cytokine-mediated downstream responses (5-7). 
The repertoire of immunological response that contributes to the pathophysiology in SA is diverse, and not well characterized. In this review, we attempt to summarize the immunobiology of airway inflammation and related molecular mechanisms that contribute to SA.

\section{Inflammatory Phenotypes Associated With Severe Asthma}

Asthma was traditionally classified as a disease with an increase in predominantly T-helper (Th) 2 cells and elevated abundance of Th2-related cytokines namely IL-4, IL-5 and IL-13. Persistence of elevated levels of these typical Th2 cytokines does not necessarily correlate with disease severity in SA (8). Current understanding indicates that the inflammatory phenotypes in SA can be Th2low as well as Th2-high (9-11). Inflammatory profile that is Th2-low with Th17-high responses, accompanied by dominant neutrophilia, is primarily defined for SA (11-14). However, some SA patients also demonstrate a Th2-high inflammation with persistent airway eosinophilia (6). Emerging studies have started to unravel the heterogeneity based on the abundance of different types of leukocytes and cytokines in the lungs and sputum of SA patients. A variety of immunophenotypes which includes Th2-low/Th17-high, Th2-high or mixed Th1/Th17 inflammatory profiles have been demonstrated in SA. There is an increase in airway granulocytes, neutrophils and eosinophils (15), with significantly higher neutrophil accumulation in the airways and sputum in patients with SA $(6,16-18)$. The percentage of neutrophils in the sputum of patients with SA is $\geq 40 \%$ higher than those with mild to moderate asthma (19). Importantly, the extent of neutrophilic inflammation has been shown to be positively associated with the severity of the disease and steroid-unresponsiveness in SA $(17,19,20)$. Th17and IL-17-mediated cellular mechanisms are primary drivers of neutrophil recruitment in the airways of patients with SA $(6,20)$. Interestingly, some patients with SA also show airway subepithelial cells expressing significantly higher IFN $\gamma$ and IL8, and lower IL-4 (Th2-low), compared to those with moderate and treatment-responsive disease, suggesting the occurrence of a Th1-skewed inflammatory disease phenotype in SA (16). Unfortunately, there are no clinically accepted biomarkers for Th2-low SA characterized with neutrophilic inflammation. Although neutrophilia is predominantly associated with SA, clinical trials with a selective CXCR2 antagonist (developed by AstraZeneca) significantly reduced neutrophil infiltrate but did not show any benefits in clinical outcomes of SA (21), further indicating that diverse leukocyte-mediated mechanisms can contribute to the disease phenotype.

SA patients with Th2-high inflammation show persistent airway eosinophilia, also termed as late-onset eosinophilic asthma (6). The eosinophil positive subtype of SA is associated with an increase in CD3+ CD4+ CD8+ T-cells, mast cells and macrophages (15). It remains unclear how this subtype of asthma patients with a Th2-high inflammatory phenotype are resistant to steroid treatments. Recent studies suggest that IL33 produced by airway epithelial cells activate innate lymphoid cells 2 (ILC2). These IL-33-activated ILC2s produce Th2cytokines IL-4, IL-5, and IL-13, leading to eosinophilic asthma which is steroid-resistant $(6,22)$. The molecular mechanisms that underpin steroid-resistance in an eosinophilic airway inflammation induced by the IL-33-ILC2 axis are not entirely defined. Nevertheless, IL-33 is significantly elevated in the airways of patients with steroid-unresponsive asthma (23-25), is known to mediate glucocorticoid resistance $(23,26)$, and being examined as a biomarker for SA (described below). Although there are several biomarkers described for Th2-high eosinophilic inflammation such as FeNO, blood total eosinophil count and eosinophil-derived neurotoxin, longitudinal cohort studies have shown that none of these biomarkers can sufficiently differentiate the phenotypes/endotypes in SA (20). Due to the diversity of inflammatory phenotypes associated with SA, it is important to unravel the immunobiology of this disease. Here, we further summarize some of the mechanisms associated with neutrophilic airway inflammation in SA.

\section{Promotion of Airway Inflammation by Neutrophils in Severe Asthma}

Neutrophils promote airway inflammation in SA by several mechanisms (Figure 1). Activated neutrophils promote dysregulation of lipid mediators of inflammation such as the ceramide/sphingosine-1-phosphate pathway, which results in further recruitment of neutrophils and eosinophils, thus amplifying airway inflammation in SA (27). Neutrophils release pro-inflammatory cytokines such as TNF and IL- $1 \beta$ that have been associated with SA $(12,28)$. Neutrophilic airway inflammation also correlates with increased expression of the NLRP3 inflammasome and IL- $1 \beta$ in SA patients $(28,29)$. A study by Kim et al showed that steroid-unresponsive neutrophilic airway inflammation is promoted by NLRP3 inflammasome, mediated primarily through the activation of caspase 1 and subsequent enhancement of IL-1 $\beta$ (29). In the study by Kim et al., inhibition of caspase 1 and suppression of IL- $1 \beta$ alleviated steroid-unresponsive neutrophilic airway inflammation in an animal model. This is corroborated by another study which showed that sputum of patients with SA have high levels of Neutrophil Extracellular Traps (NETs)-derived extracellular DNA, with concurrent activation of inflammasome marker caspase 1 in the airways (30). NETs are essentially a lattice of chromatin fibers released from activated neutrophils which contain DNA, histones, granule-derived antimicrobial peptides and enzymes such as myeloperoxidase and neutrophil elastase. Several studies have demonstrated NETs-mediated mechanisms in the enhancement of airway inflammation and subsequent airway epithelial cell damage in SA $(6,30,31)$. NETs can induce pro-inflammatory cytokines by stimulating macrophages, which further promotes neutrophil infiltration, thus generating a feedback loop for amplifying airway tissue damage in neutrophilic asthma (32). Interestingly, a NETs-independent mechanism mediated by enucleated neutrophil cytoplasts via the activation of dendritic cells, and driven by IL-17, was demonstrated in an animal model of neutrophilic airway inflammation (33). However, neutrophils, NETs and neutrophil 
cytoplasts, have all been shown to correlate with IL-17 levels in the airways, and are increased in the lungs of SA patients $(6,33)$. It thus remains unclear if NETs and/or neutrophil cytoplasts are essential for the development and persistence of SA characterized with neutrophilic airway inflammation.

\section{Neutrophil Proteins and Airway Remodeling in Severe Asthma}

Neutrophils are a major source of the proteolytic enzyme matrix metalloproteinase (MMP) 9, also known as gelatinase B, which can degrade extracellular matrix. MMP9 degrades collagen type IV of the vascular basement membrane, and promotes biological processes that contribute to the decline of lung function connected to airway hyperresponsiveness (AHR) and airway remodeling (34-36). Neutrophil-derived MMP9 is increased in the bronchoalveolar lavage fluid (BALF) and sputum of patients with SA $(37,38)$. The amount of MMP9 in BALF directly correlates with the disease severity and decline in lung function in asthma $(39,40)$. A recent study using a treatment with omalizumab, an anti-IgE monoclonal antibody, demonstrated that a decrease in BALF levels of MMP9 correlates with a lower asthma exacerbation rate in SA patients (41). Interestingly, although the decrease of MMP9 abundance in BALF correlates with reduction in reticular basement membrane thickness, it does not alter collagen or fibronectin accumulation in SA patients (41). Proteomics and transcriptomics studies have demonstrated that the neutrophil-derived protein neutrophil elastase (NE) is upregulated in the sputum of SA patients (42). NE is known to inhibit tissue inhibitors of metalloproteinases 1 (TIMP1), which is an inhibitor of MMP9, thus facilitating an increase in MMP9 and subsequent decline in lung function (43). Aligned with this, a dysregulated balance of the MMP9/TIMP1 ratio in sputum has been shown to associate with airway remodeling and asthma exacerbation $(44,45)$. Another study showed that imbalance in MMP9/TIMP1 ratio in serum associates with reduced responsiveness to steroids (46). It has been shown that ICS is ineffective in reducing MMP9 levels or controlling MMP9 activity, thus substantiating a functional role of MMP9 in steroid-unresponsiveness in SA $(47,48)$. Therefore, unraveling mechanisms that underpin MMP9 activity and subsequent airway remodeling may provide insight for the development of new intervention strategies to mitigate steroidunresponsiveness in SA. A caveat to consider is that although TIMP1 and MMP9 enzyme activities have been associated with decline in lung function, the ratio of MMP9/TIMP1 does not necessarily correlate with the disease severity in asthma (49). As MMP9 primarily promotes pulmonary fibrosis linked to airway inflammation $(35,36)$, it may well be that MMP9 may not be a critical mediator of AHR wherein the process is independent of airway inflammation. Thus, targeting MMP9 or its downstream activity may not be fully effective for the control of airway remodeling in SA. This highlights the need for a better understanding of the molecular mechanisms that underpin the biological process of airway remodeling, and that it should be considered independently from airway inflammation. This is consistent with recent studies suggesting that airway remodeling and AHR may be governed by inflammationindependent biological processes $(50,51)$.

\section{Cytokines in Steroid-Unresponsive Airway Inflammation}

The cytokine networks that facilitate and amplify the disease in SA have not yet been completely defined. Cytokines enhanced in the BALF obtained from children and adults with SA are often associated with neutrophilic inflammation, with primarily either a Th17-high or a mixed Th17/Th2/Th1 immunophenotype (17, $52,53)$. It is critical to unravel the role of specific cytokines and interacting protein partners that promote airway inflammation, AHR and the disease pathophysiology in SA, to gain a better understanding of immunomodulatory pathways that may be targeted for new interventions. Cytokines that are dominant in Th2-high inflammation such as IL-4, IL-5 and IL-13, are typically associated with eosinophil-skewed responses that are steroid-sensitive (54). However, the interplay of IL-5 with IL-33 contributes to late-onset eosinophilic asthma that is unresponsive to steroids (6). Furthermore, a recent study showed that SA patients with bacterial dysbiosis in peripheral airways have high levels of IL-13 in BALF along with neutrophilia (14). It is likely that the cytokine profile in SA is related to subclinical infections in the lungs (52). However, this is disputed by a study demonstrating that the BALF cytokine profile in neutrophilic SA is independent of respiratory pathogens (53). Thus, the association of airway cytokine profile and the microbiome in SA is not well understood, and needs to be fully examined. However, this is beyond the scope of this review. Here, we briefly discuss cytokines gaining prominence as critical mediators of airway inflammation in SA, namely IL-33, IL-17, TNF and IFN $\gamma$.

IL-33 is a member of the IL-1 family of cytokines, is a potent activator of ILC2s, and primarily promotes steroidresistant eosinophilic inflammation $(6,22,26,42)$. IL-33 is typically sequestered in the nucleus and released as an "alarmin" following cell injury and stress, as well as in response to allergen exposures. Proteases in environmental allergens can cleave the full length IL-33 to release its mature inflammatory form (55). The cleaved extracellular form of IL-33 engages the ST2 receptor to activate ILC2s, resulting in the induction of eosinophilic inflammation in response to allergens $(55,56)$. Although, many immune cells such as macrophages, dendritic cells, eosinophils and various subsets of T-cells also express the ST2 receptor (56), the interaction of IL-33 via the ST2 receptor on these immune cell types, and consequent downstream responses are not fully understood. Interestingly, chronic exposure to IL-33 results in a "memory" CD4+ Th2 cell type that preferentially produce IL5 , resulting in eosinophilic airway inflammation (56). We have recently shown that IL-33 challenge in a murine model can induce the production of IL-5 in the lungs (57), which suggests a mechanism induced by IL-33 to augment IL-5 production. Overall, the interplay of IL-33 and IL-5 signaling pathways promote eosinophilic inflammation resistant to ICS therapy in SA (7). In addition, the combinatorial effect of IL-33 with leptin, an obesity-related adipokine, promotes eosinophilic airway inflammation in obesity-related SA (58). Moreover, IL-33 can 


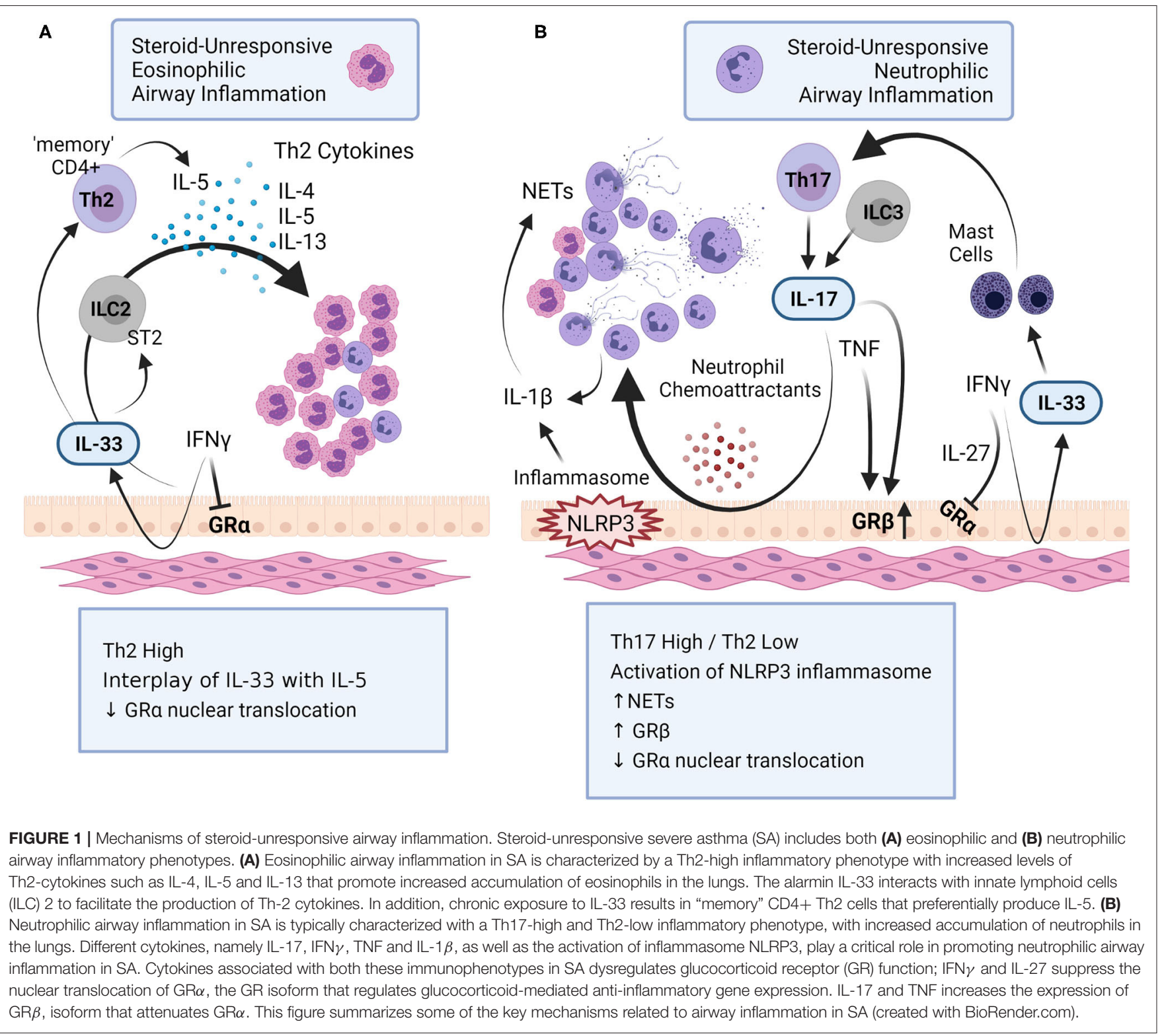

also stimulate mast cells to enhance Th17-mediated responses in neutrophilic inflammation (59). This is corroborated by various clinical studies, both in children and adults, which correlate the levels of IL-33 with asthma disease severity and steroidunresponsiveness $(23-25,60,61)$. Consequently, IL-33 is defined as a biomarker and a therapeutic target for SA $(62,63)$.

Another biomarker associated with SA is IL-17. Clinical studies show enhanced levels of IL-17, primarily IL-17A and IL-17A/F, in the lungs, serum and peripheral blood-derived mononuclear cells, of patients with SA (6, 64-67). Moreover, enhanced abundance of IL-17 in the lungs positively correlates with asthma severity, and is not mitigated by steroid treatments $(6,13,33,66,68)$. IL-17 contributes to the Th2-low/Th17high immunophenotype characterized by neutrophilic airway inflammation in SA $(6,69)$. IL-17 produced from Th17 cells recruit neutrophils to the lungs and promote steroid-resistant, neutrophilic airway inflammation (13). Similarly, IL-17 produced by ILC3 cells also leads to a steroid-resistant phenotype, which is associated with obesity-related asthma (70). Thus, both IL33 (discussed above) and IL-17 have been shown to be critical cytokines associated with promoting airway inflammation in obesity-related SA. Inflammatory processes related to obesity and asthma are thought be the underpinning bridge in obesity-related SA [reviewed in (71)]. Although obesity is associated with SA, immunomodulatory mechanisms of obesity-related SA are not completely defined.

Immunoreactive IL-17 signals through IL-17-receptors expressed on airway structural cells such as bronchial epithelial cells, resulting in the induction of neutrophil chemoattractants which enhances neutrophilic airway inflammation (69). These 
studies substantiate the critical role of IL-17 in the disease process of SA. Recent studies have shown that Th17-derived IL-17 can induce the expression of the steroid-unresponsive $\operatorname{GR} \beta$ isoform to promote steroid resistance $(66,67)$. Although, the role of IL-17 in facilitating SA is now well established, there are no effective therapies that can mitigate steroid-resistance by targeting either IL-17 or associated neutrophil signaling to effectively control SA $(72,73)$. This also point to the emerging theme that the immune networks involved in the etiology and pathophysiology of the different endotypes of SA are disparate and complex. Previous studies clearly suggest that different cytokine interactions with mixed leukocyte profiles promote various immunophenotypes in SA $(66,74)$.

Interaction of IL-17 with TNF, a Th1-effector cell cytokine, also drives Th17-inflammation and steroid-resistance, resulting in a mixed Th1/Th17-mediated response in SA $(12,75)$. TNF is elevated in the sputum, and TNF-receptors (TNFR1 and TNFR2) are enhanced in the sputum and serum of patients with SA, and are generally associated with neutrophilic inflammation (12, 76). The importance of TNF in SA is reinforced by a recent study demonstrating that treatment with azithromycin, which intervenes in TNF dysregulation, suppresses TNF and TNFR2 in SA (76). Aligned with this, a randomized clinical trial also demonstrated that azithromycin can control exacerbations in SA (77). TNF predominantly promotes airway inflammation in SA (12). However, elevated TNF in the lungs can also induce MMP9 production from bronchial epithelial cells, engaging the TNFR1-TRAF2 axis involving protein kinase C and c-Jun / Src kinase signaling pathways (78). As MMP9 promotes fibrosis and decline of lung function in SA (described above), a consequence of elevated TNF may be associated with lung remodeling in SA. Contrary to this, neutralization of TNF does not improve AHR, only improves airway inflammation, in animal model studies (12). Therefore, a direct role of TNF facilitating lung remodeling and AHR via MMP9 production cannot be definitively stated.

Synergy of different cytokines has been defined to augment steroid-resistance in asthma (Figure 1). The synergy of IFN $\gamma$ with various inflammatory mediators is described in the pathobiology of SA. For example, TNF synergizes with IFN $\gamma$ to mediate steroid-resistance in asthma (79). Integrated signaling of IFN $\gamma$ with LPS induces neutrophilic inflammation and macrophage-dependent steroid-insensitive AHR (80). Similarly, IFN $\gamma$ together with IL-27 mediate steroid-resistance and AHR in SA (81). It is thus not surprising that $\operatorname{IFN} \gamma$ is found to be increased in sputum and blood-derived cells from patients with SA compared to those with mild-tomoderate asthma (64). Similarly, high levels of IFN $\gamma$ with Th1-high immunophenotype have been demonstrated in the airways of patients with SA $(64,82)$. Another important biological function of IFN $\gamma$, in the context of $\mathrm{SA}$, is its ability to induce the production of IL-33 from airway smooth muscle and bronchial epithelial cells $(25,83)$. IL-33 is a critical mediator of the steroid-refractory phenotype (discussed above). Therefore, IFN $\gamma$ may be also contributing to the pathobiology of SA by regulating the production of IL-33 in the lungs.
Overall, studies characterizing the role of different cytokines in SA suggest that interactions of different cytokines lead to complex signaling networks resulting in disparate immunophenotypes in the disease process of SA (Figure 1). Thus, targeting a single cytokine or signaling cascade may not be effective as an intervention strategy for all SA patients. In order to gain insight into the complex signaling networks, several international consortiums have used various omics-based approaches to identify drug targets for SA (84). It is likely that common hubs or nodes within overlapping immune networks associated with the different immunophenotypes may be useful as drug targets for SA. A challenge will be to maintain the beneficial aspects of immune responses while targeting critical nodes within immune networks in order to control SA.

\section{Molecular Mechanisms of Steroid-Unresponsiveness}

Anti-inflammatory effects of glucocorticoids (type of corticosteroids) are mediated by binding to the GR isoform $\mathrm{GR} \alpha$, followed by the translocation of GR $\alpha$ from the cytoplasm to the nucleus to regulate gene transcription (6). Whereas the $\mathrm{GR} \beta$ isoform located in the nucleus does not bind to steroids, and also attenuates GR $\alpha$ function (85). A primary mechanism of steroid-resistance in SA is by the dysregulation of GR function, which can be mediated by the cytokines involved in the pathobiology of SA (discussed above). For example, a primary mode of action of TNF in SA is to promote increased expression of the GR $\beta$ isoform, which changes the GR $\alpha / \mathrm{GR} \beta$ ratio making $\mathrm{GR} \beta$ the dominant isoform thus resulting in steroid-resistance (86). Similarly, Th17-derived IL-17 induces the expression $\operatorname{GR} \beta$ to mediate steroid resistance $(66,67)$. IFN $\gamma$ together with IL-27 suppresses the nuclear translocation of GR $\alpha$ in response to glucocorticoids, to induce the steroid-refractory phenotype and AHR in SA (81). These studies clearly demonstrate that specific cytokines that are integrally associated with the various immunophenotypes of SA can facilitate dysregulation of GR-mediated response to steroids (Figure 1).

Another mechanism of anti-inflammatory effects of glucocorticoids is via the induction of a dual phosphatase, protein kinase phosphatase 1 (MKP1), which attenuates proinflammatory gene transcription by dephosphorylating p38 MAPK (87). It is important to note that the anti-inflammatory effects of MKP1 is dependent on the kinetics of its mode of action, and post-translational modifications, thus MKP1mediated functions may not be always anti-inflammatory (88). Nevertheless, the steroid-unresponsiveness phenotype is also thought to be mediated by the impairment of MKP1 function $(88,89)$. Thus, a higher concentration of steroids is required to induce MKP1 in patients with SA compared to those with steroid-sensitive disease (89). Note that critical elements of innate immunity, NLRP3 inflammasome and IL- $1 \beta$, also contribute to steroid-resistance, with NLRP3 expression enhanced in SA patients with neutrophilic inflammation $(28,29)$. TNF, which can mediate steroid-unresponsiveness (86), also regulates the expression of NLRP3 and MKP1 albeit with different kinetics (90). These studies suggest integral links 
between innate immune responses and regulatory pathways that underpin steroid-unresponsiveness, the immunobiology of which remains to be determined. Recently, epigenetic regulations primarily by microRNAs miR-9, miR-21 and miR- 126 have been defined as molecular mechanisms in the process of steroidresistance $(91,92)$. Thus, there is emerging interest in examining interventions that modulate microRNA-mediated epigenetic regulation of inflammasome and other immune pathways for the control of SA. It is clear that further studies are needed to unravel regulatory mechanisms and immune networks that control steroid-resistance, and to better understand how these are related to the various immunophenotypes in SA, in order to develop new therapies for effective management of the disease.

\section{SUMMARY}

Fundamental immunobiology of SA is extremely complex and heterogenous, with various immunophenotypes defined from patient cohorts and animal studies. The immune heterogeneity in the disease process is a considerable obstacle in developing new therapeutic approaches to efficiently mitigate SA and/or overcome steroid-resistance. It is clear that a comprehensive understanding of immune networks that contribute to the pathogenesis and regulation of inflammatory phenotypes in SA is critical to gain insight into the biological processes related to heterogeneity in SA. Furthermore, as studies emerge detailing the functions and signaling mechanisms of specific cytokines e.g., IL-17 and IL-33, or other immune mediators, on the initiation, persistence and exacerbation of SA, the utility of these immune effector elements as biomarkers or therapeutic targets will also need to be examined in the context sex and population-associated

\section{REFERENCES}

1. Nunes C, Pereira AM, Morais-Almeida M. Asthma costs and social impact. Asthma Res Pract. (2017) 3:1. doi: 10.1186/s40733-016-0029-3

2. Chung KF, Wenzel SE, Brozek JL, Bush A, Castro M, Sterk PJ, et al. International ERS/ATS guidelines on definition, evaluation and treatment of severe asthma. Eur Respir J. (2014) 43:343-73. doi: 10.1183/09031936.00202013

3. Wang E, Wechsler ME, Tran TN, Heaney LG, Jones RC, MenziesGow AN, et al. Characterization of severe asthma worldwide: data from the international severe asthma registry. Chest. (2020) 157:790804. doi: 10.1016/j.chest.2019.10.053

4. Senna G, Latorre M, Bugiani M, Caminati M, Heffler E, Morrone D, et al. Sex differences in severe asthma: results from severe asthma network in Italy-SANI. Allergy Asthma Immunol Res. (2021) 13:21928. doi: 10.4168/aair.2021.13.2.219

5. Barnes PJ, Adcock IM. Glucocorticoid resistance in inflammatory diseases. Lancet. (2009) 373:1905-17. doi: 10.1016/S0140-6736(09)60326-3

6. Nabe T. Steroid-resistant asthma and neutrophils. Biol Pharm Bull. (2020) 43:31-5. doi: 10.1248/bpb.b19-00095

7. Poon AH, Eidelman DH, Martin JG, Laprise C, Hamid Q. Pathogenesis of severe asthma. Clini Exp Allergy. (2012) 42:625-37. doi: 10.1111/j.1365-2222.2012.03983.x

8. Pelaia G, Vatrella A, Busceti MT, Gallelli L, Calabrese C, Terracciano $\mathrm{R}$, et al. Cellular mechanisms underlying eosinophilic and neutrophilic airway inflammation in asthma. Mediators Inflamm. (2015) 2015:879783. doi: 10.1155/2015/879783 immunogenetics. It may well be that combination therapies or personalized approaches will be needed for different patient groups with different disease immunophenotypes to effectively control SA. Nevertheless, detailing immune networks in SA is an unmet clinical need, which is critical for the identification of new drug targets and intervention strategies to alleviate the disease process in SA.

\section{AUTHOR CONTRIBUTIONS}

$\mathrm{CM}$ and $\mathrm{KH}$ wrote sections of the original draft. NM conceptualized the scope of the review, compiled the original draft, and extensively edited the manuscript. All authors contributed to the article and approved the submitted version.

\section{FUNDING}

Respiratory research program of NM is supported by The Canadian Institutes of Health Research (CIHR; Grant numbers, GS2-171363, PJT-155989, and SVB-158629), The Canadian Respiratory Research Network, Children's Hospital Research Institute of Manitoba, and Manitoba Workers Compensation Board. KH was supported by The University of Manitoba Vice President Undergraduate Research Award. CM is supported by Canada Graduate Scholarships - Master's Program (CGS-M).

\section{ACKNOWLEDGMENTS}

The authors thank Dr. Dustin Lippert, Grants Facilitator at The University of Manitoba, for editing the manuscript.

9. Busse WW, Kraft M, Rabe KF, Deniz Y, Rowe P, Ruddy M, Castro M. Understanding the key issues in the treatment of uncontrolled persistent asthma with type 2 inflammation. Eur Respir J. (2021) 58:2003393. doi: 10.1183/13993003.03393-2020

10. Wenzel SE. Severe adult asthmas: integrating clinical features, biology and therapeutics to improve outcomes. Am J Respir Crit Care Med. (2020) 203:809-21. doi: 10.1164/rccm.202009-3631CI

11. Wu W, Bang S, Bleecker ER, Castro M, Denlinger L, Erzurum SC, et al. Multiview cluster analysis identifies variable corticosteroid response phenotypes in severe asthma. Am J Respir Crit Care Med. (2019) 199:135867. doi: 10.1164/rccm.201808-1543OC

12. Manni ML, Trudeau JB, Scheller EV, Mandalapu S, Elloso MM, Kolls JK, et al. The complex relationship between inflammation and lung function in severe asthma. Mucosal Immunol. (2014) 7:1186-98. doi: 10.1038/mi.2014.8

13. McKinley L, Alcorn JF, Peterson A, Dupont RB, Kapadia S, Logar A, et al. TH17 cells mediate steroid-resistant airway inflammation and airway hyperresponsiveness in mice. J Immunol. (2008) 181:408997. doi: 10.4049/jimmunol.181.6.4089

14. Azim A, Green B, Lau L, Rupani H, Jayasekera N, Bruce K, et al. Peripheral airways type 2 inflammation, neutrophilia and microbial dysbiosis in severe asthma. Allergy. (2021) 76:2070-8. doi: 10.1111/all. 14732

15. Wenzel SE, Schwartz LB, Langmack EL, Halliday JL, Trudeau JB, Gibbs RL, et al. Evidence that severe asthma can be divided pathologically into two inflammatory subtypes with distinct physiologic and clinical characteristics. Am J Respir Crit Care Med. (1999) 160:1001-8. doi: 10.1164/ajrccm.160.3.9812110 
16. Shannon J, Ernst P, Yamauchi Y, Olivenstein R, Lemiere C, Foley S, et al. Differences in airway cytokine profile in severe asthma compared to moderate asthma. Chest. (2008) 133:420-6. doi: 10.1378/chest.07-1881

17. Gibson PG, Simpson JL, Saltos N. Heterogeneity of airway inflammation in persistent asthma : evidence of neutrophilic inflammation and increased sputum interleukin-8. Chest. (2001) 119:1329-36. doi: 10.1378/chest.119.5.1329

18. Wenzel SE, Balzar S, Cundall M, Chu HW. Subepithelial basement membrane immunoreactivity for matrix metalloproteinase 9: association with asthma severity, neutrophilic inflammation, and wound repair. J Allergy Clin Immunol. (2003) 111:1345-52. doi: 10.1067/mai.200 3.1464

19. Hastie AT, Moore WC, Meyers DA, Vestal PL, Li H, Peters SP, et al. Analyses of asthma severity phenotypes and inflammatory proteins in subjects stratified by sputum granulocytes. J Allergy Clin Immunol. (2010). 125:10281036. doi: 10.1016/j.jaci.2010.02.008

20. Lee Y, Quoc QL, Park H-S. Biomarkers for severe asthma: lessons from longitudinal cohort studies. Allergy Asthma Immunol Res. (2021) 13:37589. doi: 10.4168/aair.2021.13.3.375

21. Uddin M, Watz H, Malmgren A, Pedersen F. NETopathic inflammation in chronic obstructive pulmonary disease and severe asthma. Front Immunol. (2019) 10:47. doi: 10.3389/fimmu.2019.00047

22. Kabata H, Moro K, Fukunaga K, Suzuki Y, Miyata J, Masaki K, et al. Thymic stromal lymphopoietin induces corticosteroid resistance in natural helper cells during airway inflammation. Nat Commun. (2013) 4:2675. doi: $10.1038 /$ ncomms3675

23. Saglani S, Lui S, Ullmann N, Campbell GA, Sherburn RT, Mathie SA, et al. IL-33 promotes airway remodeling in pediatric patients with severe steroid-resistant asthma. J Allergy Clin Immunol. (2013) 132:67685. doi: 10.1016/j.jaci.2013.04.012

24. Mahneh SB, Movahedi M, Aryan Z, Bahar MA, Rezaei A, Sadr M, et al. Network (USERN) USE and R. Serum IL-33 Is Elevated in Children with Asthma and Is Associated with Disease Severity. IAA. (2015) 168:1936. doi: 10.1159/000442413

25. Préfontaine D, Lajoie-Kadoch S, Foley S, Audusseau S, Olivenstein R, Halayko $\mathrm{AJ}$, et al. Increased expression of IL-33 in severe asthma: evidence of expression by airway smooth muscle cells. J Immunol. (2009) 183:5094103. doi: 10.4049/jimmunol.0802387

26. Hirahara K, Mato N, Hagiwara K, Nakayama T. The pathogenicity of IL-33 on steroid-resistant eosinophilic inflammation via the activation of memory-type ST2+CD4+ T cells. J Leukoc Biol. (2018) 104:895-901. I doi: 10.1002/JLB.MR1117-456R

27. Kim S-H, Jung H-W, Kim M, Moon J-Y, Ban G-Y, Kim SJ, et al. Ceramide/sphingosine-1-phosphate imbalance is associated with distinct inflammatory phenotypes of uncontrolled asthma. Allergy. (2020) 75:19912004. doi: 10.1111/all.14236

28. Rossios C, Pavlidis S, Hoda U, Kuo C-H, Wiegman C, Russell K, et al. Sputum transcriptomics reveal upregulation of IL-1 receptor family members in patients with severe asthma. J Allergy Clin Immunol. (2018) 141:56070. doi: 10.1016/j.jaci.2017.02.045

29. Kim RY, Pinkerton JW, Essilfie AT, Robertson AAB, Baines KJ, Brown AC, et al. Role for NLRP3 inflammasome-mediated, IL-1 $\beta$-dependent responses in severe, steroid-resistant asthma. Am J Respir Crit Care Med. (2017) 196:28397. doi: 10.1164/rccm.201609-1830OC

30. Lachowicz-Scroggins ME, Dunican EM, Charbit AR, Raymond W, Looney MR, Peters MC, et al. Extracellular DNA, neutrophil extracellular traps, and inflammasome activation in severe asthma. Am J Respir Crit Care Med. (2019) 199:1076-85. doi: 10.1164/rccm.201810-1869OC

31. Hudey SN, Ledford DK, Cardet JC. Mechanisms of non-type 2 asthma. Curr Opin Immunol. (2020) 66:123-8. doi: 10.1016/j.coi.2020.10.002

32. Chen X, Li Y, Qin L, He R, Hu C. Neutrophil extracellular trapping network promotes the pathogenesis of neutrophil-associated asthma through macrophages. Immunol Invest. (2020) 50:544-61. doi: 10.1080/08820139.2020.1778720

33. Krishnamoorthy N, Douda DN, Brüggemann TR, Ricklefs I, Duvall MG, Abdulnour R-EE, et al. Neutrophil cytoplasts induce TH17 differentiation and skew inflammation toward neutrophilia in severe asthma. Sci Immunol. (2018) 3:eaao4747. doi: 10.1126/sciimmunol.aao4747
34. Lim DH, Cho JY, Miller M, McElwain K, McElwain S, Broide DH. Reduced peribronchial fibrosis in allergen-challenged MMP9-deficient mice. Am J Physiol Lung Cell Mol Physiol. (2006) 291:L265-271. doi: 10.1152/ajplung.00305.2005

35. Lagente V, Manoury B, Nénan S, Le Quément C, Martin-Chouly C, Boichot E. Role of matrix metalloproteinases in the development of airway inflammation and remodeling. Braz J Med Biol. (2005) 38:152130. doi: 10.1590/S0100-879X2005001000009

36. Corbel M, Belleguic $\mathrm{C}$, Boichot $\mathrm{E}$, Lagente $\mathrm{V}$. Involvement of gelatinases (MMP-2 and MMP-9) in the development of airway inflammation and pulmonary fibrosis. Cell Biol Toxicol. (2002) 18:51-61. doi: 10.1023/A:1014471213371

37. Barbaro MPF, Spanevello A, Palladino GP, Salerno FG, Lacedonia D, Carpagnano GE. Exhaled matrix metalloproteinase-9 (MMP-9) in different biological phenotypes of asthma. Eur J Intern Med. (2014) 25:926. doi: 10.1016/j.ejim.2013.08.705

38. Cundall M, Sun Y, Miranda C, Trudeau JB, Barnes S, Wenzel SE. Neutrophilderived matrix metalloproteinase- 9 is increased in severe asthma and poorly inhibited by glucocorticoids. J Allergy Clin Immunol. (2003) 112:106471. doi: 10.1016/j.jaci.2003.08.013

39. Farhat AA, Mohamad AS, Shareef MM, Attia GA, Eid MA, Taha RW. Asthma remodeling: The pathogenic role of matrix metalloproteinase9. Egyptian Journal of Chest Diseases and Tuberculosis. (2014) 63:7559. doi: 10.1016/j.ejcdt.2014.07.017

40. Naik SP. P A M, B S J, Madhunapantula SV, Jahromi SR, Yadav MK. Evaluation of inflammatory markers interleukin-6 (IL-6) and matrix metalloproteinase-9 (MMP-9) in asthma. J Asthma. (2017) 54:584-93. doi: 10.1080/02770903.2016.1244828

41. Zastrzezyńska W, Bazan-Socha S, Przybyszowski M, Gawlewicz-Mroczka A, Jakieła B, Plutecka $\mathrm{H}$, et al. Effect of omalizumab on bronchoalveolar lavage matrix metalloproteinases in severe allergic asthma. J Asthma. (2021) 1-11. doi: 10.1080/02770903.2021.1903917

42. Takahashi K, Pavlidis S, Ng Kee Kwong F, Hoda U, Rossios C, Sun K, et al. Sputum proteomics and airway cell transcripts of current and ex-smokers with severe asthma in U-BIOPRED: an exploratory analysis. Eur Respir J. (2018) 51:1702173. doi: 10.1183/13993003.02173-2017

43. Itoh $\mathrm{Y}$, Nagase $\mathrm{H}$. Preferential Inactivation of tissue inhibitor of metalloproteinases-1 that is bound to the precursor of matrix metalloproteinase 9 (Progelatinase B) by human neutrophil elastase *.J Biol Chem. (1995) 270:16518-21. doi: 10.1074/jbc.270.28.16518

44. Vignola AM, Riccobono L, Mirabella A, Profita M, Chanez P, Bellia V, et al. Sputum metalloproteinase-9/tissue inhibitor of metalloproteinase-1 ratio correlates with airflow obstruction in asthma and chronic bronchitis. Am J Respir Crit Care Med. (1998) 158:1945-50. doi: 10.1164/ajrccm.158.6.9803014

45. Tanaka H, Miyazaki N, Oashi K, Tanaka S, Ohmichi M, Abe S. Sputum matrix metalloproteinase-9: tissue inhibitor of metalloproteinase1 ratio in acute asthma. J Allergy Clin Immunol. (2000) 105:9005. doi: 10.1067/mai.2000.105316

46. Bossé M, Chakir J, Rouabhia M, Boulet LP, Audette M, Laviolette M. Serum matrix metalloproteinase-9:Tissue inhibitor of metalloproteinase-1 ratio correlates with steroid responsiveness in moderate to severe asthma. Am J Respir Crit Care Med. (1999) 159:596-602. doi: 10.1164/ajrccm.159.2.9802045

47. Mattos W, Lim S, Russell R, Jatakanon A, Chung KF, Barnes PJ. Matrix metalloproteinase-9 expression in asthma: effect of asthma severity, allergen challenge, and inhaled corticosteroids. Chest. (2002) 122:154352. doi: 10.1378/chest.122.5.1543

48. Grzela K, Zagórska W, Krejner A, Banaszkiewicz A, Litwiniuk M, Kulus M, et al. Inhaled corticosteroids do not reduce initial high activity of matrix metalloproteinase (MMP)-9 in exhaled breath condensates of children with asthma exacerbation: a proof of concept study. Cent Eur J Immunol. (2016) 41:221-7. doi: 10.5114/ceji.2016.60998

49. Ko FWS, Diba C, Roth M, McKay K, Johnson PRA, Salome C, et al. comparison of airway and serum matrix metalloproteinase- 9 activity among normal subjects, asthmatic patients, and patients with asthmatic mucus hypersecretion. Chest. (2005) 127:1919-27. doi: 10.1378/chest.127. 6.1919

50. Piyadasa H, Hemshekhar M, Osawa N, Lloyd D, Altieri A, Basu S, et al. Disrupting tryptophan in the central hydrophobic region selectively mitigates 
immunomodulatory activities of the innate defence regulator peptide IDR1002. J Med Chem. (2021) 64:6696-705. doi: 10.1021/acs.jmedchem.0c02065

51. Brusasco V, Crimi E, Pellegrino R. Airway hyperresponsiveness in asthma: not just a matter of airway inflammation. Thorax. (1998) 53:9928. doi: $10.1136 /$ thx. 53.11 .992

52. Alam R, Good J, Rollins D, Verma M, Chu H, Pham T-H, Martin RJ. Airway and serum biochemical correlates of refractory neutrophilic asthma. Journal of Allergy and Clinical Immunology. (2017) 140:10041014.e13. doi: 10.1016/j.jaci.2016.12.963

53. Steinke JW, Lawrence MG, Teague WG, Braciale TJ, Patrie JT, Borish L. Bronchoalveolar lavage cytokine patterns in children with severe neutrophilic and paucigranulocytic asthma. J Allergy Clini Immunol. (2021) 147:68693. doi: 10.1016/j.jaci.2020.05.039

54. Lambrecht BN, Hammad H, Fahy JV. The cytokines of asthma. Immunity. (2019) 50:975-91. doi: 10.1016/j.immuni.2019.03.018

55. Cayrol C, Duval A, Schmitt P, Roga S, Camus M, Stella A, et al. Environmental allergens induce allergic inflammation through proteolytic maturation of IL-33. Nat Immunol. (2018) 19:375-85. doi: 10.1038/s41590-018-0067-5

56. Drake LY, Kita H. IL-33: biological properties, functions, and roles in airway disease. Immunol Rev. (2017) 278:173-84. doi: 10.1111/imr.12552

57. Piyadasa H, Lloyd D, Lee AHY, Altieri A, Hemshekhar M, Osawa N, et al. Characterization of immune responses and the lung transcriptome in a murine model of IL-33 challenge. Biochim Biophys Acta Mol Basis Dis. (2020) 1866:165950. doi: 10.1016/j.bbadis.2020.165950

58. Kurokawa A, Kondo M, Arimura K, Ashino S, Tagaya E. Less airway inflammation and goblet cell metaplasia in an IL-33-induced asthma model of leptin-deficient obese mice. Respir Res. (2021) 22:166. doi: 10.1186/s12931-021-01763-3

59. Cho K-A, Suh JW, Sohn JH, Park JW, Lee H, Kang JL, et al. IL-33 induces Th17-mediated airway inflammation via mast cells in ovalbuminchallenged mice. Am J Physiol Lung Cell Mol Physiol. (2011) 302:L42940. doi: 10.1152/ajplung.00252.2011

60. Castanhinha S, Sherburn R, Walker S, Gupta A, Bossley CJ, Buckley J, et al. Pediatric severe asthma with fungal sensitization is mediated by steroid-resistant IL-33. J Allergy Clin Immunol. (2015) 136:31222. doi: 10.1016/j.jaci.2015.01.016

61. Guo Z, Wu J, Zhao J, Liu F, Chen Y, Bi L, et al. IL-33 promotes airway remodeling and is a marker of asthma disease severity. J Asthma. (2014) 51:863-9. doi: 10.3109/02770903.2014.921196

62. Li Y, Wang W, Lv Z, Li Y, Chen Y, Huang K, et al. Elevated expression of IL-33 and TSLP in the airways of human asthmatics in vivo: a potential biomarker of severe refractory disease. J Immunol. (2018) 200:225362. doi: 10.4049/jimmunol.1701455

63. Wang Y, Wang L, Hua S. Interleukin-33 in children with asthma: A systematic review and meta-analysis. Allergol Immunopathol (Madr). (2017) 45:38792. doi: 10.1016/j.aller.2016.12.007

64. Chambers ES, Nanzer AM, Pfeffer PE, Richards DF, Timms PM, Martineau AR, et al. Distinct endotypes of steroid-resistant asthma characterized by IL17Ahigh and IFN- $\gamma$ high immunophenotypes: Potential benefits of calcitriol. $J$ Allergy Clini Immunol. (2015) 136:628-37. doi: 10.1016/j.jaci.2015.01.026

65. Agache I, Ciobanu C, Agache C, Anghel M. Increased serum IL-17 is an independent risk factor for severe asthma. Respir Med. (2010) 104:11317. doi: $10.1016 /$ j.rmed.2010.02.018

66. Ramakrishnan RK, Heialy SA, Hamid Q. Role of IL-17 in asthma pathogenesis and its implications for the clinic. Expert Rev Respir Med. (2019) 13:105768. doi: 10.1080/17476348.2019.1666002

67. Vazquez-Tello A, Halwani R, Hamid Q, Al-Muhsen S. Glucocorticoid receptor-beta up-regulation and steroid resistance induction by IL-17 and IL-23 cytokine stimulation in peripheral mononuclear cells. J Clin Immunol. (2013) 33:466-78. doi: 10.1007/s10875-012-9828-3

68. Zhang Z, Biagini Myers JM, Brandt EB, Ryan PH, Lindsey M, Mintz-Cole RA, et al. $\beta$-Glucan exacerbates allergic asthma independent of fungal sensitization and promotes steroid-resistant TH2/TH17 responses. J Allergy Clin Immunol. (2017) 139:54-65. doi: 10.1016/j.jaci.2016.02.031

69. Gurczynski SJ, Moore BB. IL-17 in the lung: the good, the bad, and the ugly. Am J Physiol Lung Cell Mol Physiol. (2018) 314:L6L16. doi: 10.1152/ajplung.00344.2017

70. Kim HY, Umetsu DT, Dekruyff RH. Innate lymphoid cells in asthma: Will they take your breath away? Eur J Immunol. (2016) 46:795806. doi: $10.1002 /$ eji.201444557
71. Bantulà M, Roca-Ferrer J, Arismendi E, Picado C. Asthma and obesity: two diseases on the rise and bridged by inflammation. J Clini Med. (2021) 10:169. doi: $10.3390 / \mathrm{jcm} 10020169$

72. Seys SF, Lokwani R, Simpson JL, Bullens DMA. New insights in neutrophilic asthma. Curr Opin Pulm Med. (2019) 25:11320. doi: $10.1097 / \mathrm{MCP} .0000000000000543$

73. Olin JT, Wechsler ME. Asthma: pathogenesis and novel drugs for treatment. BMJ. (2014) 349:g5517. doi: 10.1136/bmj.g5517

74. Hastie AT, Steele C, Dunaway CW, Moore WC, Rector BM, Ampleford E, et al. Complex association patterns for inflammatory mediators in induced sputum from subjects with asthma. Clin Exp Allergy. (2018) 48:78797. doi: $10.1111 /$ cea. 13129

75. Honda K, Wada H, Nakamura M, Nakamoto K, Inui T, Sada M, et al. IL-17A synergistically stimulates TNF- $\alpha$-induced IL-8 production in human airway epithelial cells: A potential role in amplifying airway inflammation. Exp Lung Res. (2016) 42:205-16. doi: 10.1080/01902148.2016.1190796

76. Niessen NM, Gibson PG, Baines KJ, Barker D, Yang IA, Upham JW, et al. Sputum TNF markers are increased in neutrophilic and severe asthma and are reduced by azithromycin treatment. Allergy. (2021) 76:2090101. doi: $10.1111 /$ all.14768

77. Gibson PG, Yang IA, Upham JW, Reynolds PN, Hodge S, James AL, et al. Efficacy of azithromycin in severe asthma from the AMAZES randomised trial. ERJ Open Res. (2019) 5:00056-2019. doi: 10.1183/23120541.00056-2019

78. Lee I-T, Lin C-C, Wu Y-C, Yang C-M. TNF-alpha induces matrix metalloproteinase-9 expression in A549 cells: role of TNFR1/TRAF2/PKCalpha-dependent signaling pathways. J Cell Physiol. (2010) 224:454-64. doi: 10.1002/jcp.22142

79. Britt RD, Thompson MA, Sasse S, Pabelick CM, Gerber AN, Prakash YS. Th1 cytokines TNF- $\alpha$ and IFN- $\gamma$ promote corticosteroid resistance in developing human airway smooth muscle. Am J Physiol Lung Cell Mol Physiol. (2018) 316:L71-81. doi: 10.1152/ajplung.0054 7.2017

80. Yang M, Kumar RK, Foster PS. Pathogenesis of steroid-resistant airway hyperresponsiveness: interaction between IFN- $\gamma$ and TLR4/MyD88 pathways. J Immunol. (2009) 182:5107-15. doi: 10.4049/jimmunol.08 03468

81. Li JJ, Wang W, Baines KJ, Bowden NA, Hansbro PM, Gibson PG, et al. IL-27/IFN- $\gamma$ induce MyD88-dependent steroid-resistant airway hyperresponsiveness by inhibiting glucocorticoid signaling in macrophages. J Immunol. (2010) 185:4401-9. doi: 10.4049/jimmunol.1001039

82. Raundhal M, Morse C, Khare A, Oriss TB, Milosevic J, Trudeau J, et al. High IFN-gamma and low SLPI mark severe asthma in mice and humans. J Clin Invest. (2015) 125:3037-50. doi: 10.1172/JCI80911

83. Piyadasa H, Hemshekhar M, Altieri A, Basu S, van der Does AM, Halayko AJ, et al. Immunomodulatory innate defence regulator (IDR) peptide alleviates airway inflammation and hyper-responsiveness. Thorax. (2018) 73:90817. doi: 10.1136/thoraxjnl-2017-210739

84. Galeone C, Scelfo C, Bertolini F, Caminati M, Ruggiero P, Facciolongo $\mathrm{N}$, et al. Precision medicine in targeted therapies for severe asthma: is there any place for "omics" technology? Biomed Res Int. (2018) 2018:4617565. doi: 10.1155/2018/4617565

85. Oakley RH, Sar M, Cidlowski JA. The human glucocorticoid receptor beta isoform. Expression, biochemical properties, and putative function. J Biol Chem. (1996) 271:9550-9. doi: 10.1074/jbc.271.16.9550

86. Webster JC, Oakley RH, Jewell CM, Cidlowski JA. Proinflammatory cytokines regulate human glucocorticoid receptor gene expression and lead to the accumulation of the dominant negative $\beta$ isoform: A mechanism for the generation of glucocorticoid resistance. Proc Natl Acad Sci U S A. (2001) 98:6865-70. doi: 10.1073/pnas.121455098

87. Desmet SJ, De Bosscher K. Glucocorticoid receptors: finding the middle ground. J Clin Invest. (2017) 127:1136-45. doi: 10.1172/JCI88886

88. Hoppstädter J, Ammit AJ. Role of dual-specificity phosphatase 1 in glucocorticoid-driven anti-inflammatory responses. Front Immunol. (2019) 10:1446. doi: 10.3389/fimmu.2019.01446

89. Wang $\mathrm{M}$, Gao $\mathrm{P}$, Wu $\mathrm{X}$, Chen $\mathrm{Y}$, Feng $\mathrm{Y}$, Yang $\mathrm{Q}$, et al Impaired anti-inflammatory action of glucocorticoid in neutrophil from patients with steroid-resistant asthma. Respir Res. (2016) 17:153. doi: 10.1186/s12931-016-0462-0

90. Prabhala P, Bunge K. Rahman MdM, Ge Q, Clark AR, Ammit AJ. Temporal regulation of cytokine mRNA expression by tristetraprolin: dynamic control 
by p38 MAPK and MKP-1. Am J Physiol Lung Cell Mol Physiol. (2015) 308:L973-80. doi: 10.1152/ajplung.00219.2014

91. Wadhwa R, Dua K, Adcock IM, Horvat JC, Kim RY, Hansbro PM. Cellular mechanisms underlying steroid-resistant asthma. Eur Respir Rev. (2019) 28:190096. doi: 10.1183/16000617.009 6-2019

92. Mei D, Tan WSD, Wong WSF. Pharmacological strategies to regain steroid sensitivity in severe asthma and COPD. Curr Opin Pharmacol. (2019) 46:7381. doi: 10.1016/j.coph.2019.04.010

Conflict of Interest: The authors declare that the research was conducted in the absence of any commercial or financial relationships that could be construed as a potential conflict of interest.
Publisher's Note: All claims expressed in this article are solely those of the authors and do not necessarily represent those of their affiliated organizations, or those of the publisher, the editors and the reviewers. Any product that may be evaluated in this article, or claim that may be made by its manufacturer, is not guaranteed or endorsed by the publisher.

Copyright (C) 2021 Marshall, Hasani and Mookherjee. This is an open-access article distributed under the terms of the Creative Commons Attribution License (CC BY).

The use, distribution or reproduction in other forums is permitted, provided the original author(s) and the copyright owner(s) are credited and that the original publication in this journal is cited, in accordance with accepted academic practice. No use, distribution or reproduction is permitted which does not comply with these terms. 